\title{
Ruptured Globe
}

National Cancer Institute

\section{Source}

National Cancer Institute. Ruptured Globe. NCI Thesaurus. Code C118871.

Full thickness wound of the eyewall (cornea and/or sclera) due to blunt trauma. (adapted from the Birmingham Eye Trauma Terminology System (BETTS)) 